\title{
ANKLE REFLEX TIMING AS A SCREENING PROCEDURE FOR THE DETECTION OF HYPOTHYROIDISM
}

\author{
J. C. MUCKLOW AND D. J. P. BARKER, M.R.C.P., Ph.D., B.Sc. \\ Department of Medicine, University of Birmingham, Queen Elizabeth Hospital, Birmingham 15
}

MEASUREMENT of the ankle reflex time by means of a kinemometer has been shown to be a useful aid in the clinical diagnosis of hypothyroidism (Abraham, Atkinson, and Roscoe, 1966). It is not known whether it will also serve as a screening procedure for this disorder. In the Birmingham region a follow-up system has recently been initiated to effect the continued surveillance of thyrotoxic patients treated with radioactive iodine (Barker and Bishop, 1969). These patients require periodic screening for hypothyroidism. Before the follow-up system was devised, a preliminary study was made on a group of patients treated with ${ }^{131}$ I from 6 to 14 years previously. As part of this study the ankle reflex times of the patients were measured.

\section{METHOD}

The reflex times were recorded by means of a recent modification of the kinemometer first used by Lawson (1958). The patient kneels on a chair and a horseshoe magnet is strapped to the sole of the foot. When the ankle reflex is elicited movement of the magnet induces a current in twin induction coils positioned 2 to $4 \mathrm{~cm}$. away. The two leads from the coils are connected directly to leads from a portable electrocardiograph. The connection is such that movement of the magnet towards the coil results in a downward deflection on the E.C.G. trace.

The tracing obtained consists of an initial rapid oscillation, produced by the hammer striking the tendon, followed by a slower downward defiection caused by muscle contraction, and an upward deflection representing relaxation. The distance measured as the 'reflex time' is that between the maximum rate of contraction (V) and the maximum rate of relaxation $(\mathrm{P})$, those being the two points most sharply defined on all traces. The mean VP time of at least three traces is obtained and the resulting figure is rounded to the nearest $20 \mathrm{msec}$.

The configuration of the trace varies with the direction of movement of the magnet in relation to the coil, and careful positioning of both magnet and coil is required to obtain uniformity of the traces. The initial positions of both magnet and coil must be adjusted so that the plane passing through both poles of the magnet remains parallel to the plane of the coil throughout the reflex movement. This adjustment may take some time to achieve, and in some cases may prove impossible; but a slight variation in the shape of the trace does not always preclude accurate measurement of the VP interval.

In the present study it was found that the VP time was independent of the amplitude of the trace and also of the frequency at which reflexes were elicited. It has previously been recommended that a long recovery period should be allowed between reflexes? but this was not found to be necessary.

\section{Results}

Reflex times were recorded in 225 patients, of whom 46 were shown to be hypothyroid and to require thyroxine. The diagnosis of hypothyroidism depended on full clinical assessment, measurement of the serum protein bound iodine and cholesterol levels, the results of radio-iodine uptake studies where necessary, and the response to thyroxine therapy. There is a considerable overlap between the distributions of reflex times in hypothyroid and euthyroid patients (Figure). The reflex times of

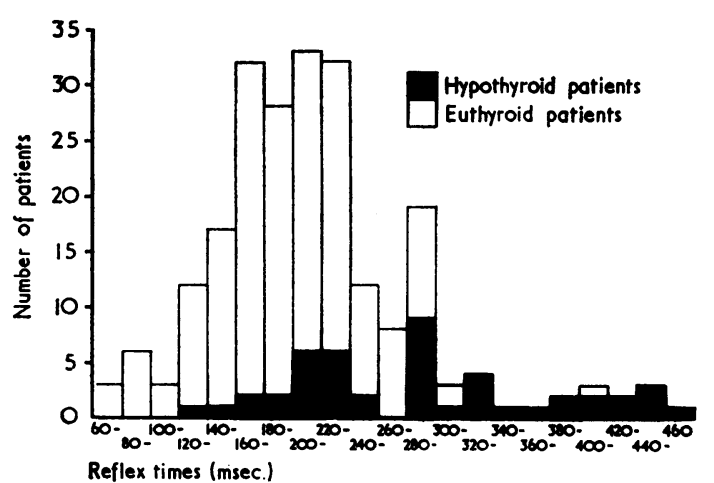

FIGURB-Distribution of ankle reflex times in 225 patients. 
hypothyroid patients ranged from 120 to 460 msec., while those of euthyroid patients ranged from 60 to $400 \mathrm{msec}$. Only 3 out of 20 patients with reflex times of 300 msec. or longer were euthyroid; and it is interesting that the single euthyroid patient with a very prolonged time of $400 \mathrm{msec}$. also had a low serum P.B.I. $(2 \cdot 6 \mu \mathrm{g} . / 100 \mathrm{ml}$.) but had no clinical evidence of hypothyroidism.

\section{Discussion}

Nordyke and Gilbert (1967) used a photomotogram to screen for hypothyroidism among more than 2,000 people attending a Health Fair. They found a considerable overlap between the reflex times of euthyroid and hypothyroid individuals. They concluded that in order to use ankle reflex timing, by itself, as a procedure for screening populations it would be necessary to set an upper limit of normal which, while restricting the number of false positives, could result in the incorrect classification of the majority of cases of mild hypothyroidism, and half of those with moderate hypothyroidism. They suggested that the number of false positives would be reduced if screening was confined to certain selected groups, such as those who have had thyroid surgery or radioactive iodine therapy. The results of the present study show that measurement of the ankle reflex time would not be an effective screening procedure for the detection of hypothyroidism among patients treated with ${ }^{131} \mathrm{I}$. If reflex times are defined as abnormal when they exceed some limiting value, determined by reference to the distribution shown in the Figure, it is not possible to select a value which would yield an acceptable number of false negatives and positives. Although in the present study a kinemometer, rather than a photomotogram, was used, previous studies carried out on hospital patients have shown no marked differences between the results obtained with these two instruments (Nuki and Bayliss, 1968).
The findings in this study support the use of the kinemometer as a diagnostic aid in clinical practice. If the reflex time exceeds the limit of 300 msec., suggested by Abraham et al. (1966), it is likely that the patient has hypothyroidism. If the time is between 200 and $300 \mathrm{msec}$. no conclusions can be drawn, but hypothyroidism is unlikely when the time is less than $200 \mathrm{msec}$. If reflex times were compared with previous recordings from the same patient this might provide a sensitive index of the development of hypothyroidism, but there is no evidence to confirm this.

\section{SUMmaRY}

A kinemometer was used to measure the ankle reflex times in a group of patients who had received radio-iodine therapy for thyrotoxicosis. It was concluded that ankle reflex timing was not an effective screening procedure for the detection of hypothyroidism.

It is a pleasure to thank Dr. A. S. Abraham, who lent us a kinemometer and advised us on its use.

\section{REFERENCES}

Abraham, A. S., Atkinson, M., and Roscoe, B. (1966). Value of ankle-jerk timing in the assessment of thyroid function. Brit. med. J., 1, 830.

BARKer, D. J. P., and Bishop, J. M. (1969). A computer based screening system for patients at risk of hypothyroidism. Lancet, 2, 835.

LAwson, J. D. (1958). The free Achilles reflex in hypothroidism and hyperthyroidism. New Engl. J. Med., 259, 761.

NORDYKE, R. A., and GLLBERT, F. I. (1967). Mass screening for hypothyroidism by the Achilles reflex time. Arch. environm. Hlth, 14, 827.

NuKI, G., and BayLiss, R. I. S. (1968). The Achilles tendon reflex as an index of thyroid function. Postgrad. med. J., 44, 97. 